\title{
Supergraph calculation of one-loop divergences in higher-derivative $6 D$ SYM theory
}

\author{
I.L. Buchbinder, ${ }^{a, b, c}$ E.A. Ivanov, ${ }^{c}$ B.S. Merzlikin ${ }^{d, a, c}$ and K.V. Stepanyantz ${ }^{e, c}$ \\ ${ }^{a}$ Department of Theoretical Physics, Tomsk State Pedagogical University, \\ 634061, Tomsk, Russia \\ ${ }^{b}$ National Research Tomsk State University, \\ 634050, Tomsk, Russia \\ ${ }^{c}$ Bogoliubov Laboratory of Theoretical Physics, JINR, \\ 141980 Dubna, Moscow region, Russia \\ ${ }^{d}$ Tomsk State University of Control Systems and Radioelectronics, \\ 634050 Tomsk, Russia \\ ${ }^{e}$ Department of Theoretical Physics, Moscow State University, \\ 119991, Moscow, Russia \\ E-mail: joseph@tspu.edu.ru, eivanov@theor.jinr.ru, \\ merzlikin@tspu.edu.ru, stepan@m9com.ru
}

ABSTRACT: We apply the harmonic superspace approach for calculating the divergent part of the one-loop effective action of renormalizable $6 D, \mathcal{N}=(1,0)$ supersymmetric higherderivative gauge theory with a dimensionless coupling constant. Our consideration uses the background superfield method allowing to carry out the analysis of the effective action in a manifestly gauge covariant and $\mathcal{N}=(1,0)$ supersymmetric way. We exploit the regularization by dimensional reduction, in which the divergences are absorbed into a renormalization of the coupling constant. Having the expression for the one-loop divergences, we calculate the relevant $\beta$-function. Its sign is specified by the overall sign of the classical action which in higher-derivative theories is not fixed a priori. The result agrees with the earlier calculations in the component approach. The superfield calculation is simpler and provides possibilities for various generalizations.

Keywords: Field Theories in Higher Dimensions, Renormalization Regularization and Renormalons, Superspaces, Supersymmetric Gauge Theory

ARXiv EPRINT: 2004.12657 


\section{Contents}

1 Introduction 1

2 Harmonic superspace formulation of $6 D, \mathcal{N}=(1,0)$ higher-derivative SYM theory

3 Background field quantization in harmonic superspace 5

4 Calculation of the divergent supergraphs $\quad 6$

5 Conclusion $\quad 9$

A The second variation of the classical action 9

B Divergences of the supergraphs with a gauge loop 12

\section{Introduction}

It is well known that field theories with standard kinetic terms and standard interactions are not renormalizable in higher dimensions because of the dimensionful coupling constants and the too slow decrease of propagators at large momenta. Supersymmetry is sometimes capable to improve the ultraviolet behavior in the lowest loops [1-4], but does not lead to the renormalizability [4] even in theories with maximally extended supersymmetry [5-8].

A way to obtain a higher-dimensional renormalizable theory is to allow the action to include terms with higher derivatives. The study of the higher derivative theories at the classical and quantum levels has a long history that apparently begins with the seminal work by Pais and Uhlenbeck [9]. Although the higher-derivative theories suffer from the ghost states in the spectrum, they still attract much attention and are widely used in gravity, cosmology and quantum field theory (see, e.g., [10-14]). Some of the recent applications, as well as the discussion of ways to evade the problem of ghosts, can be found in refs. [15-20] and reviews [21, 22] (and the references therein). So, the higher-derivative models are considered to be very interesting and deserving the study from different points of view.

An important example of application of the higher-derivative theories is the regularization by higher-order covariant derivatives [23, 24]. This regularization is self-consistent and, for supersymmetric theories, can be formulated in a manifestly supersymmetric way [25-28] consistent with the non-renormalization theorems (see, e.g., [29-31]). When applied for investigating quantum corrections in supersymmetric field theories, the higher-derivative regularization allowed to reveal some interesting features of them (see, e.g., [32, 33] and references therein). This is one more argument why it is useful to study the quantum corrections in higher-derivative supersymmetric theories in various dimensions. 
In this paper we consider the six-dimensional higher-derivative supersymmetric gauge theory proposed in ref. [34] and calculate the divergent part of the one-loop effective action, using the regularization by dimensional reduction. This theory describes the following set of the interacting $6 D$ fields: the vector field, the Weyl spinor field, and three real scalar fields, all being in the adjoint representation of the gauge group. In the gauge field sector the action starts with the term containing four derivatives

$$
-\frac{1}{g_{0}^{2}} \operatorname{tr} \int d^{6} x\left(\nabla^{M} F_{M N}\right)^{2}
$$

where $F_{M N}$ is the standard Yang-Mills strength. This implies that the coupling constant $g_{0}$ is dimensionless. The manifestly supersymmetric formulation of the theory in $6 D$, $\mathcal{N}=(1,0)$ harmonic superspace and the full off-shell component form of the action in the Wess-Zumino gauge were earlier given in ref. [34]. ${ }^{1}$

In the case under consideration, the issue of renormalizabity was studied in [34] (see also [35]) in a component formulation and in [36] in a superfield formalism. The actual calculations definitely show that the theory is renormalizable at one loop. ${ }^{2}$ The one-loop divergences, renormalization of the coupling constant and the corresponding beta-function in this theory were calculated in refs. $[34,35]$ by two different methods based on the component formulations.

The study of various aspects of the four-dimensional supersymmetric quantum field theories (see, e.g., the monographs [29-31]) provided an evidence that the most attractive and elegant way of investigating their quantum properties is by using superfield methods. The superfield formulation of $6 D$ supersymmetric theories was constructed in refs. [38-40] in terms of $6 D, \mathcal{N}=(1,0)$ harmonic superspace which is quite similar to its $4 D, \mathcal{N}=2$ prototype [41-43]. The main advantage of such a formulation is the possibility to keep manifest $\mathcal{N}=(1,0)$ supersymmetry at all steps of quantum calculations. In our recent papers [44-50] we developed the harmonic superfield approach for calculating the lowest off-shell quantum corrections in various $6 D, \mathcal{N}=(1,0)$ and $\mathcal{N}=(1,1)$ supersymmetric theories. In the present paper we apply this superfield technique for studying the one-loop effective action in $6 D, \mathcal{N}=(1,0)$ higher-derivative gauge theory of ref. [34].

The paper is organized as follows. In section 2 we collect the basic notions of $6 D$, $\mathcal{N}=(1,0)$ harmonic superspace and the formulation of the model under consideration within its framework. Section 3 presents the manifestly supersymmetric and gauge covariant quantization of this theory and the construction of the corresponding effective action. In section 4 we compute the one-loop divergences by a direct calculation of the harmonic supergraphs and find the $\beta$-function. The results and some further problems are summarized in Conclusion. The technical details of the calculation are contained in appendices A and B.

\footnotetext{
${ }^{1}$ The radical difference of such a higher-derivative theory from the theories regularized by higher-order derivatives is that the actions of the latter theories involve higher-derivative terms as corrections to the standard kinetic terms with canonical numbers of derivatives (two for bosons and one for fermions), while in the case under consideration no canonical kinetic terms are present from the very beginning.

${ }^{2}$ The theory under consideration possesses a chiral anomaly [37] which, in principle, can violate the renormalizability. However it is known that the anomaly does not affect the form of one-loop divergences.
} 


\section{Harmonic superspace formulation of $6 D, \mathcal{N}=(1,0)$ higher-derivative SYM theory}

The harmonic superspace technique is most convenient for formulating theories with $6 D$, $\mathcal{N}=(1,0)$ supersymmetry as it suggests the manifestly supersymmetric and gauge invariant scheme of their quantization.

In our notation the coordinates of $6 D$ Minkowski space and the $\mathcal{N}=(1,0)$ Grassmann coordinates are denoted by $x^{M}$ and $\theta^{a i}$, with $M=0, \ldots, 5, a=1, \ldots, 4$, and $i=1,2$. The coordinates $\left(x^{M}, \theta^{a i}, u_{i}^{ \pm}\right)$of the harmonic superspace include in addition the harmonic variables $u_{i}^{ \pm}$which obey the constraints $u^{+i} u_{i}^{-}=1, u_{i}^{-} \equiv\left(u^{+i}\right)^{*}$. Having these coordinates at hand, one can construct the harmonic derivatives

$$
D^{++}=u^{+i} \frac{\partial}{\partial u^{-i}} ; \quad D^{--}=u^{-i} \frac{\partial}{\partial u^{+i}} ; \quad D^{0}=u^{+i} \frac{\partial}{\partial u^{+i}}-u^{-i} \frac{\partial}{\partial u^{-i}},
$$

which generate an $\mathrm{SU}(2)$ algebra. The harmonic superspace contains an analytic subspace closed under the $6 D, \mathcal{N}=(1,0)$ supersymmetry transformations. It is parametrized by the coordinates

$$
x_{A}^{M}=x^{M}+\frac{i}{2} \theta^{-} \gamma^{M} \theta^{+} ; \quad \theta^{ \pm a}=u_{i}^{ \pm} \theta^{a i},
$$

where $\gamma^{M}$ are $6 D \gamma$-matrices.

We also introduce the harmonic spinor covariant derivatives

$$
D_{a}^{+}=u_{i}^{+} D_{a}^{i} ; \quad D_{a}^{-}=u_{i}^{-} D_{a}^{i},
$$

the only non-zero anticommutation relation among which being $\left\{D_{a}^{+}, D_{b}^{-}\right\}=i\left(\gamma^{M}\right)_{a b} \partial_{M}$. Due to the anticommutativity of the derivatives $D_{a}^{+}$any product of four such derivatives (defined with respect to the same harmonic variable $u$ ) is reduced to the expression

$$
\left(D^{+}\right)^{4}=-\frac{1}{24} \varepsilon^{a b c d} D_{a}^{+} D_{b}^{+} D_{c}^{+} D_{d}^{+} .
$$

In this paper we adopt the following convention for the superspace integration measures needed for constructing $\mathcal{N}=(1,0)$ invariant actions:

$$
\int d \zeta^{(-4)} \equiv \int d^{6} x d^{4} \theta^{+} ; \quad \int d^{14} z \equiv \int d^{6} x d^{8} \theta=\int d^{6} x d^{4} \theta^{+}\left(D^{+}\right)^{4} .
$$

In the harmonic superspace formulation the gauge field is carried by the superfield $V^{++}(z, u)=V^{++A} t^{A}$ which obeys the analyticity condition

$$
D_{a}^{+} V^{++}=0
$$

and is real with respect to a generalized conjugation denoted by a tilde, $\widetilde{V^{++}}=V^{++}$. In this paper we use the Hermitian generators $t^{A}$ which are normalized by the conditions $\operatorname{tr}\left(t^{A} t^{B}\right)=\delta^{A B} / 2$. From the gauge superfield $V^{++}$one can construct a non-analytic superfield

$$
V^{--}(z, u) \equiv \sum_{n=1}^{\infty}(-i)^{n+1} \int d u_{1} d u_{2} \ldots d u_{n} \frac{V^{++}\left(z, u_{1}\right) V^{++}\left(z, u_{2}\right) \ldots V^{++}\left(z, u_{n}\right)}{\left(u^{+} u_{1}^{+}\right)\left(u_{1}^{+} u_{2}^{+}\right) \ldots\left(u_{n}^{+} u^{+}\right)},
$$


and, further, the harmonic gauge superfield strength

$$
F^{++} \equiv\left(D^{+}\right)^{4} V^{--} \text {. }
$$

It is evidently analytic. Moreover, it satisfies the off-shell condition

$$
\nabla^{++} F^{++} \equiv D^{++} F^{++}+i\left[V^{++}, F^{++}\right]=0,
$$

which is a consequence of the harmonic flatness condition

$$
D^{++} V^{--}-D^{--} V^{++}+i\left[V^{++}, V^{--}\right]=0 .
$$

The latter can be considered as a definition of $V^{--}$.

The gauge transformations in the harmonic superspace are parametrized by a real (with respect to the tilde-conjugation) analytic superfield $\lambda=\lambda^{A} t^{A}$ :

$$
V^{ \pm \pm} \rightarrow e^{i \lambda} V^{ \pm \pm} e^{-i \lambda}-i e^{i \lambda} D^{ \pm \pm} e^{-i \lambda}, \quad F^{++} \rightarrow e^{i \lambda} F^{++} e^{-i \lambda} .
$$

The $6 D, \mathcal{N}=(1,0)$ supersymmetric generalization of the usual $6 D$ Yang-Mills theory in the harmonic superspace formulation is given by the action [51]

$$
S_{\mathrm{SYM}}=\frac{1}{f_{0}^{2}} \sum_{n=2}^{\infty} \frac{(-i)^{n}}{n} \operatorname{tr} \int d^{14} z d u_{1} \ldots d u_{n} \frac{V^{++}\left(z, u_{1}\right) \ldots V^{++}\left(z, u_{n}\right)}{\left(u_{1}^{+} u_{2}^{+}\right) \ldots\left(u_{n}^{+} u_{1}^{+}\right)},
$$

with the coupling constant $f_{0}$ having the dimension of the inverse mass. It is clear that this theory is not renormalizable by power counting. The one-loop divergences for this theory have been calculated in ref. [46].

In the present paper we will consider a different theory which contains the higher (four) derivatives. Unlike the second-order derivative SYM theory with the action (2.12), the higher-derivative theory we are considering is characterized by a dimensionless coupling constant. Such a theory was formulated in harmonic $6 D$ superspace in [34]. It is described by the following manifestly gauge invariant and $\mathcal{N}=(1,0)$ supersymmetric action

$$
S= \pm \frac{1}{2 g_{0}^{2}} \operatorname{tr} \int d \zeta^{(-4)} d u\left(F^{++}\right)^{2}= \pm \frac{1}{4 g_{0}^{2}} \int d \zeta^{(-4)} d u\left(F^{++A}\right)^{2},
$$

where the analytic harmonic superfield strength $F^{++}$is defined in eq. (2.8). The aim of our paper is to investigate the one-loop divergences for the theory with the action (2.13).

The sign of the action (2.13) deserves some comments. In conventional field theory models without higher derivatives the overall sign is fixed by the requirement that the energy is positive. In the higher-derivative theories the energy is not positively defined in general. This means, that there are no actual reasons to fix an overall sign of the action in such theories. This is why we cannot fix the sign of the action (2.13). ${ }^{3}$ Note that in ref. [34] there was chosen the sign minus (corresponding to the sign in (1.1)) since it gives rise to the correct sign in front of the component kinetic term of the triplet of scalar fields entering the gauge $\mathcal{N}=(1,0)$ multiplet (the former auxiliary fields). However, this does not imply the positivity of energy for all component fields which involve higher-derivative ghosts for any sign. In ref. [35] there was chosen the sign "plus". In order to have a freedom to compare our results with those obtained in refs. [34, 35], we prefer not to fix the overall sign of the action.

\footnotetext{
${ }^{3}$ This point was also noted in [35].
} 


\section{Background field quantization in harmonic superspace}

The harmonic superspace technique makes it possible to construct the manifestly $\mathcal{N}=$ $(1,0)$ supersymmetric quantization procedure. It is also convenient to use the background superfield method [52-54] which provides a manifestly gauge invariant effective action. In $6 D, \mathcal{N}=(1,0)$ harmonic superspace it is formulated similarly to the $4 D, \mathcal{N}=2$ case treated in $[55,56]$. In particular, the background-quantum splitting is linear,

$$
V^{++}=\boldsymbol{V}^{++}+v^{++}
$$

where $\boldsymbol{V}^{++}$and $v^{++}$are the background and quantum gauge superfields, respectively. After the substitution of (3.1) in the action (2.13) the gauge invariance (2.11) amounts to the two types of transformations. The background gauge invariance

$$
\boldsymbol{V}^{++} \rightarrow e^{i \lambda} \boldsymbol{V}^{++} e^{-i \lambda}-i e^{i \lambda} D^{++} e^{-i \lambda} ; \quad v^{++} \rightarrow e^{i \lambda} v^{++} e^{-i \lambda}
$$

remains a manifest symmetry of the effective action, while the quantum gauge invariance

$$
\boldsymbol{V}^{++} \rightarrow \boldsymbol{V}^{++} ; \quad v^{++} \rightarrow e^{i \lambda}\left(v^{++}+\boldsymbol{V}^{++}\right) e^{-i \lambda}-\boldsymbol{V}^{++}-i e^{i \lambda} D^{++} e^{-i \lambda}
$$

is broken by the gauge-fixing procedure down to the invariance under the BRST transformations. It is assumed that the gauge-fixing term should be chosen invariant under the transformations (3.2). The harmonic superspace analog of the $\xi$-gauge is then given by the action

$$
S_{\mathrm{gf}}=\mp \frac{1}{2 g_{0}^{2} \xi_{0}} \operatorname{tr} \int d^{14} z d u_{1} d u_{2} \frac{\left(u_{1}^{-} u_{2}^{-}\right)}{\left(u_{1}^{+} u_{2}^{+}\right)^{3}} e^{i \boldsymbol{b}_{1}} e^{-i \boldsymbol{b}_{2}}\left(\boldsymbol{\nabla}_{2}^{++} v_{2}^{++}\right) e^{i \boldsymbol{b}_{2}} e^{-i \boldsymbol{b}_{1}} \widehat{\square}_{1}\left(\boldsymbol{\nabla}_{1}^{++} v_{1}^{++}\right),
$$

where the operator

$$
\widehat{\square} \equiv \frac{1}{2}\left(D^{+}\right)^{4}\left(\nabla^{--}\right)^{2}
$$

is reduced to the covariant analog of the d'Alambertian operator, when acting on analytic superfields. ${ }^{4}$ The background covariant derivatives are defined as

$$
\boldsymbol{\nabla}^{++}=D^{++}+i \boldsymbol{V}^{++} ; \quad \nabla^{--}=D^{--}+i \boldsymbol{V}^{--} .
$$

Evidently, if they act on a superfield in the adjoint representation (e.g., on $v^{++}$), the gauge superfields should be expanded over the generators of the adjoint representation, so that

$$
\boldsymbol{\nabla}^{ \pm \pm} v^{++}=D^{ \pm \pm} v^{++}+i\left[\boldsymbol{V}^{ \pm \pm}, v^{++}\right]
$$

The background bridge superfield $\boldsymbol{b}$ in eq. (3.4) is related to the background superfields $\boldsymbol{V}^{++}$and $\boldsymbol{V}^{--}$(constructed out of $\boldsymbol{V}^{++}$by the equation similar to (2.7)) via the relations

$$
\boldsymbol{V}^{++}=-i e^{i \boldsymbol{b}} D^{++} e^{-i \boldsymbol{b}} ; \quad \boldsymbol{V}^{--}=-i e^{i \boldsymbol{b}} D^{--} e^{-i \boldsymbol{b}} .
$$

\footnotetext{
${ }^{4}$ In principle, in the framework of the background field method we could use any appropriate gauge preserving the background gauge invariance. However, it is technically convenient to choose the action $S_{\mathrm{gf}}$ to be of the same degree in derivatives as the classical action.
} 
The subscripts 1 and 2 in eq. (3.4) refer to the harmonic variables $u_{1}$ or $u_{2}$ present in $S_{\mathrm{gf}}$. Similar notation will be used below.

The action for the Faddeev-Popov ghosts corresponding to the gauge-fixing action (3.4) is obtained in the standard way (see, e.g., [55]) and is given by

$$
S_{\mathrm{FP}}=\operatorname{tr} \int d \zeta^{(-4)} d u b \nabla^{++}\left(\nabla^{++} c+i\left[v^{++}, c\right]\right),
$$

where the anticommuting analytic superfields $b$ and $c$ stand for the Faddeev-Popov antighosts and ghosts, respectively. However, the presence of the operator $\widehat{\square}$ in eq. (3.4) change the form of the Nielsen-Kallosh determinant. Namely, in the case under consideration it can be written in the form

$$
\Delta_{\mathrm{NK}}=\operatorname{Det}^{-1 / 2}\left(\boldsymbol{\nabla}^{++}\right)^{2} \operatorname{Det} \widehat{\square}=\int D \varphi D \chi^{(+4)} D \sigma \exp \left(i S_{\mathrm{NK}}\right),
$$

where the set of Nielsen-Kallosh ghosts involves the commuting analytic superfield $\varphi$ together with the Grassmann-odd analytic superfields $\chi^{(+4)}$ and $\sigma$, all being in the adjoint representation of the gauge group. The action for these ghosts reads

$$
S_{\mathrm{NK}}=\operatorname{tr} \int d \zeta^{(-4)} d u\left(-\frac{1}{2}\left(\boldsymbol{\nabla}^{++} \varphi\right)^{2}+\chi^{(+4)} \widehat{\square} \sigma\right) .
$$

Then the generating functional for the considered theory can finally be written in the form

$Z\left[\right.$ Sources, $\left.\boldsymbol{V}^{++}\right]=\int D v^{++} D b D c D \varphi D \chi^{(+4)} D \sigma \exp \left\{i\left(S+S_{\mathrm{gf}}+S_{\mathrm{FP}}+S_{\mathrm{NK}}+S_{\text {sources }}\right)\right\}$.

The source term is defined as

$$
S_{\text {sources }}=\int d \zeta^{(-4)} d u J^{++A} v^{++A}+\ldots,
$$

where dots denote terms corresponding to various ghost superfields. The effective action $\Gamma\left[\right.$ Fields, $\left.\boldsymbol{V}^{++}\right]$is defined as the Legendre transform of the generating functional for the connected Green functions $W \equiv-i \ln Z$. Setting all quantum fields equal to zero, we obtain the manifestly gauge invariant action $\Gamma\left[\boldsymbol{V}^{++}\right] \equiv \Gamma\left[\right.$ Fields $\left.\rightarrow 0, \boldsymbol{V}^{++}\right]$.

Like in the case of standard $6 D, \mathcal{N}=(1,0)$ SYM theory $[45,46]$, there are two ways to calculate divergent terms in this action. One of them is based on the superfield proper-time technique and preserves manifest gauge invariance at all steps. Another goes through the direct calculation of the relevant Feynman supergraphs with invoking gauge invariance at the final stage. Here we employ the second method, leaving the first one for the future study.

\section{Calculation of the divergent supergraphs}

The one-loop contribution to the two-point Green function of the background gauge superfield for the considered model is given by the supergraphs depicted on figure 1 . We will calculate it in the minimal gauge $\xi=1$, where $\xi$ is the renormalized gauge-fixing parameter. The external wavy lines in the supergraphs on figure 1 represent the background gauge 

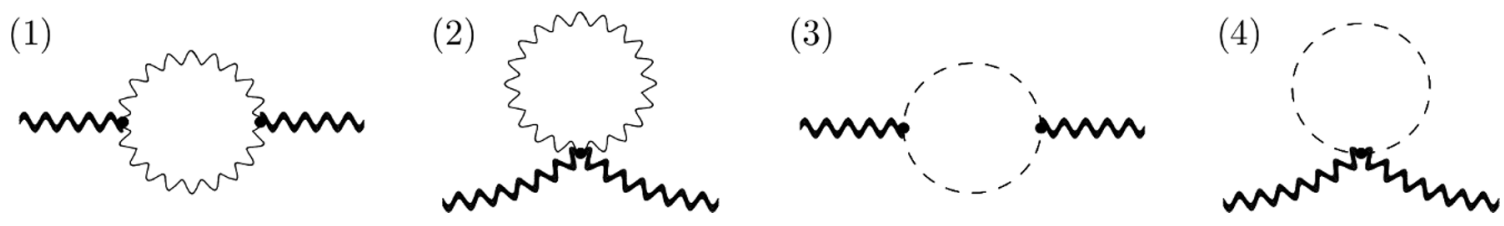

Figure 1. Supergraphs which allow to calculate the divergent part of the one-loop effective action for the model (2.13). The dashed lines stand for all ghost superfields present in the theory.

superfield $\boldsymbol{V}^{++}$(or the background bridge $\boldsymbol{b}$ ). The wavy internal lines correspond to the propagators of the quantum gauge superfield $v^{++}$. In the Feynman gauge this propagator has the simplest form and is given by the expression

$$
-\left.\frac{\delta^{2} Z_{0}}{\delta J_{1}^{++A} \delta J_{2}^{++B}}\right|_{J^{++}=0}= \pm 2 i g_{0}^{2} \frac{1}{\partial^{4}}\left(D_{2}^{+}\right)^{4} \delta^{14}\left(z_{1}-z_{2}\right) \delta^{(2,-2)}\left(u_{1}, u_{2}\right),
$$

where

$$
\delta^{14}\left(z_{1}-z_{2}\right) \equiv \delta^{6}\left(x_{1}-x_{2}\right) \delta^{8}\left(\theta_{1}-\theta_{2}\right)
$$

and $Z_{0}$ denotes the generating functional of the free theory. The dashed lines denote propagators of the Faddeev-Popov and Nielsen-Kallosh ghosts. All ghost contributions have already been calculated earlier [45, 46]. Actually, the ghost part of the generating functional for the theory under consideration and for the theory (2.12) differ only in the expression

$$
\left(\int D \chi^{(+4)} D \sigma \exp \left\{i \operatorname{tr} \int d \zeta^{(-4)} d u \chi^{(+4)} \widehat{\square} \sigma\right\}\right)^{1 / 2} .
$$

However, according to [46] this expression cannot produce divergences. This implies that the total ghost contribution (which include both Faddeev-Popov and Nielsen-Kallosh parts and can be found by calculating the supergraphs (3) and (4) in figure 1) coincides with the one for the theory (2.12) and, according to [46], is equal to

$$
\left(\Delta \Gamma_{\infty}^{(1)}\right)_{\text {ghost }}=\frac{C_{2}}{3 \varepsilon(4 \pi)^{3}} \operatorname{tr} \int d \zeta^{(-4)} d u\left(\boldsymbol{F}^{++}\right)^{2}
$$

where it is assumed that the regularization by dimensional reduction is used, with $\varepsilon \equiv 6-D$. The constant $C_{2}$ is defined by the equation $f^{A C D} f^{B C D}=C_{2} \delta^{A B}$, where the structure constants $f^{A B C}$ are given by the commutator of generators, $\left[t^{A}, t^{B}\right]=i f^{A B C} t^{C}$.

Thus, it remains to calculate only the one-loop divergences produced by the supergraphs containing a loop of the quantum gauge superfield. These divergences are completely determined by the part of the total action quadratic in the quantum gauge superfield (which does not contain other quantum superfields). Obviously, such terms are present only in the classical action (2.13) and the gauge-fixing action (3.4). In appendix A we demonstrate 
that, in the Feynman gauge $\xi_{0}=1,{ }^{5}$

$$
\begin{aligned}
S^{(2)}+S_{\mathrm{gf}}= & \pm \frac{1}{2 g_{0}^{2}} \operatorname{tr} \int d \zeta^{(-4)} d u v^{++} \widehat{\square}^{2} v^{++} \\
& \mp \frac{i}{2 g_{0}^{2}} \operatorname{tr} \int d^{14} z d u_{1} d u_{2} \frac{1}{\left(u_{1}^{+} u_{2}^{+}\right)^{2}} e^{i \boldsymbol{b}_{1}} e^{-i \boldsymbol{b}_{2}} v_{2}^{++} e^{i \boldsymbol{b}_{2}} e^{-i \boldsymbol{b}_{1}}\left[\boldsymbol{F}_{1}^{++}, \nabla_{1}^{--} v_{1}^{++}\right] .
\end{aligned}
$$

Starting from this expression we calculate the one-loop divergences coming from the supergraphs which contain a loop of the quantum gauge superfield (see appendix B). Namely, from the expression (4.5) we find the vertices in the supergraphs depicted on figure 1. Next, we calculate the first two supergraphs in figure 1 and obtain the corresponding contribution to the divergent part of the two-point Green function of the background gauge superfield. Finally, taking into account the background gauge invariance of the effective action, we obtain the general result for all one-loop divergences coming from the supergraphs with a loop of the quantum gauge superfield inside. The result is given by the expression

$$
\left(\Delta \Gamma_{\infty}^{(1)}\right)_{\text {gauge }}=-\frac{4 C_{2}}{\varepsilon(4 \pi)^{3}} \operatorname{tr} \int d \zeta^{(-4)} d u\left(\boldsymbol{F}^{++}\right)^{2} .
$$

Summing up eqs. (4.4) and (4.6), we obtain that the divergent part of the one-loop effective action for the theory (2.13) regularized by the dimensional reduction takes the form

$$
\Delta \Gamma_{\infty}^{(1)}=\left(\Delta \Gamma_{\infty}^{(1)}\right)_{\text {gauge }}+\left(\Delta \Gamma_{\infty}^{(1)}\right)_{\text {ghost }}=-\frac{11 C_{2}}{3 \varepsilon(4 \pi)^{3}} \operatorname{tr} \int d \zeta^{(-4)} d u\left(\boldsymbol{F}^{++}\right)^{2} .
$$

This result exactly agrees with the ones obtained in refs. [34, 35] starting from the component formulation of the theory in the Wess-Zumino gauge.

Adding the expression (4.7) to the classical action (2.13) we can find quantum corrections to the coupling constant $g_{0}$,

$$
\begin{aligned}
\Gamma-S_{\mathrm{gf}}= & \left( \pm \frac{1}{2 g_{0}^{2}}-\frac{11 C_{2}}{3 \varepsilon(4 \pi)^{3}}\right) \operatorname{tr} \int d \zeta^{(-4)} d u\left(\boldsymbol{F}^{++}\right)^{2} \\
& + \text { finite one-loop contributions + higher order corrections. }
\end{aligned}
$$

From this expression we see that the renormalized coupling constant $g$ is related to $g_{0}$ as

$$
\frac{1}{g^{2}}=\frac{1}{g_{0}^{2}} \mp \frac{22 C_{2}}{3 \varepsilon(4 \pi)^{3}}+\text { higher orders, }
$$

which exactly agrees with the relation obtained in (the revised version of) ref. [34] and in ref. [35]. It is more convenient to rewrite it in terms of $\alpha \equiv g^{2} / 4 \pi$,

$$
\frac{1}{\alpha}=\frac{1}{\alpha_{0}} \mp \frac{22 C_{2}}{3 \varepsilon(4 \pi)^{2}}+\text { higher orders. }
$$

This relation implies that the one-loop $\beta$-function is given by the expression

$$
\beta(\alpha)=\mp \frac{11 \alpha^{2} C_{2}}{24 \pi^{2}}+\text { higher orders. }
$$

Thus, the lower sign corresponds to the Landau zero, while the upper sign corresponds to the asymptotic freedom. If one takes into account the difference in the notations, the former choice was used in ref. [34], while the calculation of ref. [35] corresponds to the latter case.

\footnotetext{
${ }^{5}$ When calculating the one-loop divergences, there is no difference between choices $\xi_{0}=1$ and $\xi=1$.
} 


\section{Conclusion}

In this paper we have considered the higher-derivative $6 D, \mathcal{N}=(1,0)$ supersymmetric Yang-Mills theory in the harmonic superspace formulation. The theory was quantized within the background superfield method which allows to preserve the manifest gauge invariance and $6 D, \mathcal{N}=(1,0)$ supersymmetry at all stages of the quantum calculations. Using the supergraph technique and the regularization by dimensional reduction we have calculated the one-loop divergences of the quantum effective action. The calculations were organized as follows. The divergences were firstly found to the lowest order with respect to the background gauge superfield. Then, using the manifest gauge invariance, the full result for the divergent part of the one-loop effective action was restored. ${ }^{6}$ It was shown that the divergences can be absorbed into the renormalization of the dimensionless coupling constant $g_{0}$. The corresponding $\beta$-function was computed and its sign was shown to be determined by the sign of the initial classical action.

It is natural to expect that the theory will remain renormalizable after adding to the action $(2.13)$ the action of $6 D, \mathcal{N}=(1,0)$ gauge theory without higher derivatives involving a dimensionful coupling constant $f_{0}$. It would be interesting to study the renormalization properties of such a theory involving two coupling constants $g_{0}$ and $f_{0}$. Another interesting problem is the construction of the $6 D, \mathcal{N}=(1,1)$ higher-derivative supersymmetric gauge theory and the study of its renormalization properties. For this purpose one should extend the theory considered here by higher-derivative couplings with the hypermultiplet in the adjoint representation (e.g., along the line of ref. [57]) and try to ensure the hid$\operatorname{den} \mathcal{N}=(0,1)$ supersymmetry in such an extended $6 D$ system. These problems will be addressed elsewhere.

\section{Acknowledgments}

I.L.B. and E.A.I. are grateful to Andrei Smilga for useful discussions in the course of this work. The work was supported by Russian Science Foundation, grant No. 16-12-10306.

\section{A The second variation of the classical action}

For calculating the one-loop divergences we need to single out that part of the action which is quadratic in the quantum gauge superfields. As is evident from eq. (3.1), to this end one should calculate the second variation of the action, then make the replacement

$$
\delta V^{++} \rightarrow v^{++} ; \quad V^{++} \rightarrow \boldsymbol{V}^{++}
$$

and finally multiply the result by $1 / 2$.

The first variation of the action (2.13) is given by the expression

$$
\delta S= \pm \frac{1}{g_{0}^{2}} \operatorname{tr} \int d \zeta^{(-4)} d u F^{++} \delta F^{++}= \pm \frac{1}{g_{0}^{2}} \operatorname{tr} \int d^{14} z d u F^{++} \delta V^{--} .
$$

\footnotetext{
${ }^{6}$ As was already mentioned, an alternative to this calculation is the computational procedure preserving the manifest gauge invariance at all steps and based on the superfield proper-time technique [45]. We plan to consider this approach in the forthcoming paper.
} 
To obtain the variation $\delta V^{--}$, note that, as a consequence of eq. (2.10), it should satisfy the relation

$$
\nabla^{++} \delta V^{--}=\nabla^{--} \delta V^{++}
$$

where the covariant derivatives are defined as

$$
\nabla^{++} \equiv D^{++}+i V^{++}, \quad \nabla^{--} \equiv D^{--}+i V^{--}
$$

Note that, in contrast to eqs. (3.6), these expressions contain the superfields $V^{ \pm \pm}$(instead of $\boldsymbol{V}^{ \pm \pm}$). The solution of eq. (A.3) satisfies the equation

$$
\delta V^{--}=\frac{1}{2}\left(\nabla^{--}\right)^{2} \delta V^{++}-\frac{1}{2} \nabla^{++} \nabla^{--} \delta V^{--} .
$$

Substituting this expression into (A.2) and using (2.9), the first variation of the action can be presented in the form

$$
\delta S= \pm \frac{1}{2 g_{0}^{2}} \operatorname{tr} \int d^{14} z d u F^{++}\left(\nabla^{--}\right)^{2} \delta V^{++} .
$$

Next, we calculate the second variation

$$
\begin{gathered}
\delta^{2} S=\delta(\delta S)= \pm \frac{1}{2 g_{0}^{2}} \operatorname{tr} \int d^{14} z d u\left(\delta F^{++}\left(\nabla^{--}\right)^{2} \delta V^{++}+i F^{++}\left[\delta V^{--}, \nabla^{--} \delta V^{++}\right]\right. \\
\left.+i F^{++} \nabla^{--}\left[\delta V^{--}, \delta V^{++}\right]\right) .
\end{gathered}
$$

Substituting $\delta F^{++}=\left(D^{+}\right)^{4} \delta V^{--}$into this expression, after some algebra we obtain

$$
\begin{aligned}
\delta^{2} S= \pm \frac{1}{g_{0}^{2}} \operatorname{tr} \int d^{14} z d u\left(\delta V^{--} \widehat{\square} \delta V^{++}\right. & \\
& \left.-\frac{i}{2} \delta V^{--}\left[F^{++}, \nabla^{--} \delta V^{++}\right]+\frac{i}{2} \delta V^{--}\left[\nabla^{--} F^{++}, \delta V^{++}\right]\right),
\end{aligned}
$$

where

$$
\widehat{\square} \equiv \frac{1}{2}\left(D^{+}\right)^{4}\left(\nabla^{--}\right)^{2} .
$$

To find the variation $\delta V^{--}$we introduce the (non-analytic) bridge superfield $b$ related to $V^{ \pm \pm}$by the relations similar to (3.8),

$$
V^{++}=-i e^{i b} D^{++} e^{-i b} ; \quad V^{--}=-i e^{i b} D^{--} e^{-i b} .
$$

Then, taking into account that $\nabla^{ \pm \pm}=e^{i b} D^{ \pm \pm} e^{-i b}$, the solution of eq. (A.3) can be written as [58]

$$
\delta V_{1}^{--}=\int d u_{2} \frac{1}{\left(u_{1}^{+} u_{2}^{+}\right)^{2}} e^{i b_{1}} e^{-i b_{2}} \delta V_{2}^{++} e^{i b_{2}} e^{-i b_{1}},
$$

where the subscripts refer to the corresponding harmonic variables. 
Substituting the expression (A.11) into eq. (A.8) we rewrite the second variation of the action (2.13) as

$$
\begin{aligned}
\delta^{2} S= \pm \frac{1}{g_{0}^{2}} \operatorname{tr} \int & d^{14} z d u_{1} d u_{2} \frac{1}{\left(u_{1}^{+} u_{2}^{+}\right)^{2}} e^{i b_{1}} e^{-i b_{2}} \delta V_{2}^{++} e^{i b_{2}} e^{-i b_{1}} \\
& \times\left(\widehat{\square}_{1} \delta V_{1}^{++}-\frac{i}{2}\left[F_{1}^{++}, \nabla_{1}^{--} \delta V_{1}^{++}\right]+\frac{i}{2}\left[\nabla_{1}^{--} F_{1}^{++}, \delta V_{1}^{++}\right]\right) .
\end{aligned}
$$

Making in this expression the replacement (A.1) and multiplying the result by $1 / 2$ we obtain that part of the action (2.13) which is quadratic in the quantum gauge superfields,

$$
\begin{aligned}
S^{(2)}= \pm \frac{1}{2 g_{0}^{2}} \operatorname{tr} & \int d^{14} z d u_{1} d u_{2} \frac{1}{\left(u_{1}^{+} u_{2}^{+}\right)^{2}} e^{i \boldsymbol{b}_{1}} e^{-i \boldsymbol{b}_{2}} v_{2}^{++} e^{i \boldsymbol{b}_{2}} e^{-i \boldsymbol{b}_{1}} \\
& \times\left(\widehat{\square}_{1} v_{1}^{++}-\frac{i}{2}\left[\boldsymbol{F}_{1}^{++}, \boldsymbol{\nabla}_{1}^{--} v_{1}^{++}\right]+\frac{i}{2}\left[\boldsymbol{\nabla}_{1}^{--} \boldsymbol{F}_{1}^{++}, v_{1}^{++}\right]\right) .
\end{aligned}
$$

To obtain the analogous part of the total action, we should add the gauge-fixing action (3.4) to this expression. Using the identity

$$
\begin{aligned}
{\left[\widehat{\square}, \boldsymbol{\nabla}^{++}\right] v^{++} } & =\frac{1}{2}\left(D^{+}\right)^{4}\left[\left(\boldsymbol{\nabla}^{--}\right)^{2}, \boldsymbol{\nabla}^{++}\right] v^{++}=-\frac{1}{2}\left(D^{+}\right)^{4}\left(D^{0} \boldsymbol{\nabla}^{--}+\boldsymbol{\nabla}^{--} D^{0}\right) v^{++} \\
& =-\left(D^{+}\right)^{4} \boldsymbol{\nabla}^{--} v^{++}=-i\left[\boldsymbol{F}^{++}, v^{++}\right]
\end{aligned}
$$

after integrating by parts with respect to the harmonic derivatives the gauge-fixing term (3.4) can be rewritten as

$$
\begin{aligned}
S_{\mathrm{gf}}=\mp \frac{1}{2 g_{0}^{2} \xi_{0}} \operatorname{tr} \int & d^{14} z d u_{1} d u_{2} e^{i \boldsymbol{b}_{1}} e^{-i \boldsymbol{b}_{2}} v_{2}^{++} e^{i \boldsymbol{b}_{2}} e^{-i \boldsymbol{b}_{1}}\left\{D_{1}^{++} D_{2}^{++}\left(\frac{\left(u_{1}^{-} u_{2}^{-}\right)}{\left(u_{1}^{+} u_{2}^{+}\right)^{3}}\right) \widehat{\square}_{1} v_{1}^{++}\right. \\
& \left.+i D_{2}^{++}\left(\frac{\left(u_{1}^{-} u_{2}^{-}\right)}{\left(u_{1}^{+} u_{2}^{+}\right)^{3}}\right)\left[\boldsymbol{F}_{1}^{++}, v_{1}^{++}\right]\right\} .
\end{aligned}
$$

Then, with the help of the identities

$$
\begin{aligned}
D_{1}^{++} D_{2}^{++}\left(\frac{\left(u_{1}^{-} u_{2}^{-}\right)}{\left(u_{1}^{+} u_{2}^{+}\right)^{3}}\right) & =\frac{1}{\left(u_{1}^{+} u_{2}^{+}\right)^{2}}-\frac{1}{2}\left(D_{1}^{--}\right)^{2} \delta^{(2,-2)}\left(u_{1}, u_{2}\right), \\
D_{2}^{++}\left(\frac{\left(u_{1}^{-} u_{2}^{-}\right)}{\left(u_{1}^{+} u_{2}^{+}\right)^{3}}\right) & =\frac{\left(u_{1}^{-} u_{2}^{+}\right)}{\left(u_{1}^{+} u_{2}^{+}\right)^{3}}=-\frac{1}{2} D_{1}^{--}\left(\frac{1}{\left(u_{1}^{+} u_{2}^{+}\right)^{2}}\right)
\end{aligned}
$$

it is convenient to rearrange the expression (A.15) to the form

$$
\begin{aligned}
S_{\mathrm{gf}}= & \pm \frac{1}{2 g_{0}^{2} \xi_{0}} \operatorname{tr} \int d \zeta^{(-4)} d u v^{++} \widehat{\square}^{2} v^{++} \mp \frac{1}{2 g_{0}^{2} \xi_{0}} \operatorname{tr} \int d^{14} z d u_{1} d u_{2} \frac{1}{\left(u_{1}^{+} u_{2}^{+}\right)^{2}} \\
& \times e^{i \boldsymbol{b}_{1}} e^{-i \boldsymbol{b}_{2}} v_{2}^{++} e^{i \boldsymbol{b}_{2}} e^{-i \boldsymbol{b}_{1}}\left\{\widehat{\square}_{1} v_{1}^{++}+\frac{i}{2} \boldsymbol{\nabla}_{1}^{--}\left[\boldsymbol{F}_{1}^{++}, v_{1}^{++}\right]\right\} .
\end{aligned}
$$


The quadratic in the quantum gauge superfield part of the total action is obtained as a sum of the expressions (A.13) and (A.18),

$$
\begin{aligned}
& S^{(2)}+S_{\mathrm{gf}}= \pm \frac{1}{2 g_{0}^{2} \xi_{0}} \operatorname{tr} \int d \zeta^{(-4)} d u v^{++} \widehat{\square}^{2} v^{++} \pm \frac{1}{2 g_{0}^{2}} \operatorname{tr} \int d^{14} z d u_{1} d u_{2} \frac{1}{\left(u_{1}^{+} u_{2}^{+}\right)^{2}} e^{i \boldsymbol{b}_{1}} e^{-i \boldsymbol{b}_{2}} v_{2}^{++} \\
& \times e^{i \boldsymbol{b}_{2}} e^{-i \boldsymbol{b}_{1}}\left\{\left(1-\frac{1}{\xi_{0}}\right) \widehat{\square}_{1} v_{1}^{++}+\frac{i}{2}\left(1-\frac{1}{\xi_{0}}\right)\left[\boldsymbol{\nabla}_{1}^{--} \boldsymbol{F}_{1}^{++}, v_{1}^{++}\right]-\frac{i}{2}\left(1+\frac{1}{\xi_{0}}\right)\left[\boldsymbol{F}_{1}^{++}, \boldsymbol{\nabla}_{1}^{--} v_{1}^{++}\right]\right\} .
\end{aligned}
$$

This expression is drastically simplified in the Feynman gauge $\xi_{0}=1$. In this gauge eq. (A.19) is reduced to the expression (4.5).

\section{B Divergences of the supergraphs with a gauge loop}

Let us calculate the one-loop divergences coming from the first two supergraphs presented in figure 1. Both these supergraphs contain a loop of the quantum gauge superfield, so that the vertices can be found from the expression (4.5). In particular, the triple vertex present in the supergraph (1) can be written as

$$
\begin{aligned}
& \mp \frac{1}{4 g_{0}^{2}} f^{A B C} \int d^{14} z d u \partial^{2} v^{++A}\left[\boldsymbol{V}_{\text {linear }}^{--B} D^{--} v^{++C}+D^{--}\left(\boldsymbol{V}_{\text {linear }}^{--B} v^{++C}\right)\right] \\
& \pm \frac{1}{4 g_{0}^{2}} f^{A B C} \int d^{14} z d u_{1} d u_{2} \frac{1}{\left(u_{1}^{+} u_{2}^{+}\right)^{2}} v_{2}^{++A} \boldsymbol{F}_{\text {linear }, 1}^{++B} D_{1}^{--} v_{1}^{++C} \equiv \operatorname{Ver}_{1}+\operatorname{Ver}_{2}
\end{aligned}
$$

where the subscript "linear" means that it is enough to consider only the part linear in the background gauge superfield $\boldsymbol{V}^{++}$,

$$
\boldsymbol{V}_{\text {linear }, 1}^{--A}=\int d u_{2} \frac{1}{\left(u_{1}^{+} u_{2}^{+}\right)^{2}} \boldsymbol{V}_{2}^{++A} ; \quad \boldsymbol{F}_{\text {linear }, 1}^{++A}=\int d u_{2} \frac{1}{\left(u_{1}^{+} u_{2}^{+}\right)^{2}}\left(D_{1}^{+}\right)^{4} \boldsymbol{V}_{2}^{++A} .
$$

We see that the vertex (B.1) can naturally be divided into the two parts, $\mathrm{Ver}_{1}$ and $\mathrm{Ver}_{2}$. The first one is composed of the terms which include $\boldsymbol{V}_{\text {linear }}^{--B}$, while the second one contains the term with $\boldsymbol{F}_{\text {linear }}^{++B}$. Therefore, the supergraph (1) in figure 1 splits into three subgraphs, namely, $\mathrm{Ver}_{1}-\mathrm{Ver}_{1}, \mathrm{Ver}_{2}-\mathrm{Ver}_{2}$, and $\mathrm{Ver}_{1}-\mathrm{Ver}_{2}$. The subgraph containing two vertices $\mathrm{Ver}_{1}$ is very similar to an analogous supergraph calculated in ref. [46] and vanishes due to the presence of the factors

$$
\left.\left(D_{1}^{--}\right)^{2}\left(u_{1}^{+} u_{2}^{+}\right)^{4}\right|_{u_{1}=u_{2}}=0 \quad \text { or }\left.\quad D_{1}^{--} D_{2}^{--}\left(u_{1}^{+} u_{2}^{+}\right)^{4}\right|_{u_{1}=u_{2}}=0 .
$$

The subgraph $\mathrm{Ver}_{2}-\mathrm{Ver}_{2}$ is proportional to

$$
\int \frac{d^{6} k}{(2 \pi)^{6}} \frac{1}{k^{4}(k+p)^{4}}
$$

and is, therefore, finite. This implies that this subgraph does not contribute to the divergent part of the one-loop effective action and can be omitted.

Thus, we see that the only non-trivial contribution of the supergraph (1) to the oneloop divergences can appear from the (logarithmically divergent) subgraph $\mathrm{Ver}_{1}-\mathrm{Ver}_{2}$. 
Applying Feynman rules, we find that the considered contribution to the effective action is given by the expression

$$
\begin{aligned}
& \text { Ver }_{1}-\operatorname{Ver}_{2}=-\frac{i C_{2}}{4} \int d^{14} z_{1} d^{14} z_{2} d u_{1} d u_{2} d u_{3} \frac{1}{\left(u_{2}^{+} u_{3}^{+}\right)^{2}} \boldsymbol{V}_{\text {linear }}^{--A}\left(z_{1}, u_{1}\right) \boldsymbol{F}_{\text {linear }}^{++A}\left(z_{2}, u_{2}\right) \\
& \times\left\{D_{2}^{--}\left[\frac{1}{\partial^{4}}\left(D_{2}^{+}\right)^{4} \delta^{14}\left(z_{1}-z_{2}\right) \delta^{(2,-2)}\left(u_{1}, u_{2}\right)\right] D_{1}^{--}\left[\frac{1}{\partial^{2}}\left(D_{3}^{+}\right)^{4} \delta^{14}\left(z_{1}-z_{2}\right) \delta^{(2,-2)}\left(u_{1}, u_{3}\right)\right]\right. \\
& -D_{1}^{--} D_{2}^{--}\left[\frac{1}{\partial^{4}}\left(D_{2}^{+}\right)^{4} \delta^{14}\left(z_{1}-z_{2}\right) \delta^{(2,-2)}\left(u_{1}, u_{2}\right)\right] \frac{1}{\partial^{2}}\left(D_{3}^{+}\right)^{4} \delta^{14}\left(z_{1}-z_{2}\right) \delta^{(2,-2)}\left(u_{1}, u_{3}\right) \\
& +D_{2}^{--}\left[\frac{1}{\partial^{2}}\left(D_{2}^{+}\right)^{4} \delta^{14}\left(z_{1}-z_{2}\right) \delta^{(2,-2)}\left(u_{1}, u_{2}\right)\right] D_{1}^{--}\left[\frac{1}{\partial^{4}}\left(D_{3}^{+}\right)^{4} \delta^{14}\left(z_{1}-z_{2}\right) \delta^{(2,-2)}\left(u_{1}, u_{3}\right)\right] \\
& \left.-D_{1}^{--} D_{2}^{--}\left[\frac{1}{\partial^{2}}\left(D_{2}^{+}\right)^{4} \delta^{14}\left(z_{1}-z_{2}\right) \delta^{(2,-2)}\left(u_{1}, u_{2}\right)\right] \frac{1}{\partial^{4}}\left(D_{3}^{+}\right)^{4} \delta^{14}\left(z_{1}-z_{2}\right) \delta^{(2,-2)}\left(u_{1}, u_{3}\right)\right\}
\end{aligned}
$$

Note that, without a loss of generality, we can assume that all spinor derivatives act on the point $z_{1}$.

The $\delta$-functions $\delta^{14}\left(z_{1}-z_{2}\right)$ include $\delta^{8}\left(\theta_{1}-\theta_{2}\right)$, and the product of two Grassmannian $\delta$-functions does not vanish only in the situation when at least 8 spinor covariant derivatives act on them. Taking into account this property and using the identity

$$
\delta^{8}\left(\theta_{1}-\theta_{2}\right)\left(D_{1}^{+}\right)^{4}\left(D_{2}^{+}\right)^{4} \delta^{8}\left(\theta_{1}-\theta_{2}\right)=\left(u_{1}^{+} u_{2}^{+}\right)^{4} \delta^{8}\left(\theta_{1}-\theta_{2}\right)
$$

we can do one of the integrals over $d^{8} \theta$. Then, in the momentum representation, eq. (B.5) can be brought in the form

$$
\begin{aligned}
& \frac{i C_{2}}{2} \int d^{8} \theta d u_{1} d u_{2} d u_{3} \int \frac{d^{6} p}{(2 \pi)^{6}} \boldsymbol{V}_{\text {linear }}^{--A}\left(-p, \theta, u_{1}\right) \boldsymbol{F}_{\text {linear }}^{++A}\left(p, \theta, u_{2}\right) \frac{1}{\left(u_{2}^{+} u_{3}^{+}\right)^{2}} \\
& \quad \times \int \frac{d^{6} k}{(2 \pi)^{6}} \frac{1}{k^{4}(k+p)^{2}}\left\{D_{2}^{--}\left[\left(u_{2}^{+} u_{3}^{+}\right)^{4} \delta^{(2,-2)}\left(u_{1}, u_{2}\right)\right] D_{1}^{--} \delta^{(2,-2)}\left(u_{1}, u_{3}\right)\right. \\
& \left.\quad-D_{1}^{--} D_{2}^{--}\left[\left(u_{2}^{+} u_{3}^{+}\right)^{4} \delta^{(2,-2)}\left(u_{1}, u_{2}\right)\right] \delta^{(2,-2)}\left(u_{1}, u_{3}\right)\right\} .
\end{aligned}
$$

After integrating by parts with respect to the derivative $D_{1}^{--}$in the last term, this expression can be rewritten as

$$
\begin{aligned}
& \frac{i C_{2}}{2} \int d^{8} \theta d u_{1} d u_{2} d u_{3} \int \frac{d^{6} p}{(2 \pi)^{6}} \frac{d^{6} k}{(2 \pi)^{6}} \frac{1}{k^{4}(k+p)^{2}} \boldsymbol{F}_{\text {linear }}^{++A}\left(p, \theta, u_{2}\right)\left\{\frac{2}{\left(u_{2}^{+} u_{3}^{+}\right)^{2}}\right. \\
& \quad \times \boldsymbol{V}_{\text {linear }}^{--A}\left(-p, \theta, u_{1}\right) D_{2}^{--}\left[\left(u_{2}^{+} u_{3}^{+}\right)^{4} \delta^{(2,-2)}\left(u_{1}, u_{2}\right)\right] D_{1}^{--} \delta^{(2,-2)}\left(u_{1}, u_{3}\right)+\left(u_{2}^{+} u_{3}^{+}\right)^{2} \\
& \left.\quad \times D_{1}^{--} \boldsymbol{V}_{\text {linear }}^{--A}\left(-p, \theta, u_{1}\right) D_{2}^{--} \delta^{(2,-2)}\left(u_{1}, u_{2}\right) \delta^{(2,-2)}\left(u_{1}, u_{3}\right)\right\}
\end{aligned}
$$

where we took into account that

$$
\left(u_{2}^{+} u_{3}^{+}\right) \delta^{(2,-2)}\left(u_{1}, u_{2}\right) \delta^{(2,-2)}\left(u_{1}, u_{3}\right) \rightarrow 0
$$


Once again, integrating by parts with respect to the derivative $D_{2}^{--}$and doing the integral over $d u_{1}$ with the help of $\delta^{(2,-2)}\left(u_{1}, u_{2}\right)$, we obtain

$$
\begin{aligned}
\operatorname{Ver}_{1}-\text { Ver }_{2}= & \frac{i C_{2}}{2} \int d^{8} \theta d u_{2} d u_{3} \int \frac{d^{6} p}{(2 \pi)^{6}} \frac{d^{6} k}{(2 \pi)^{6}} \frac{1}{k^{4}(k+p)^{2}} \\
& \times\left\{4 \boldsymbol{V}_{\text {linear }}^{--A}\left(-p, \theta, u_{2}\right) \boldsymbol{F}_{\text {linear }}^{++A}\left(p, \theta, u_{2}\right)\left(u_{2}^{-} u_{3}^{+}\right)\left(u_{2}^{+} u_{3}^{+}\right) D_{2}^{--} \delta^{(2,-2)}\left(u_{2}, u_{3}\right)\right. \\
& -2 \boldsymbol{V}_{\text {linear }}^{--A}\left(-p, \theta, u_{2}\right) D_{2}^{--} \boldsymbol{F}_{\text {linear }}^{++A}\left(p, \theta, u_{2}\right)\left(u_{2}^{+} u_{3}^{+}\right)^{2} D_{2}^{--} \delta^{(2,-2)}\left(u_{2}, u_{3}\right) \\
& -2 D_{2}^{--} \boldsymbol{V}_{\text {linear }}^{--A}\left(-p, \theta, u_{2}\right) \boldsymbol{F}_{\text {linear }}^{++A}\left(p, \theta, u_{2}\right)\left(u_{2}^{-} u_{3}^{+}\right)\left(u_{2}^{+} u_{3}^{+}\right) \delta^{(2,-2)}\left(u_{2}, u_{3}\right) \\
& \left.-D_{2}^{--} \boldsymbol{V}_{\text {linear }}^{--A}\left(-p, \theta, u_{2}\right) D_{2}^{--} \boldsymbol{F}_{\text {linear }}^{++A}\left(p, \theta, u_{2}\right)\left(u_{2}^{+} u_{3}^{+}\right)^{2} \delta^{(2,-2)}\left(u_{2}, u_{3}\right)\right\} .
\end{aligned}
$$

Two last terms in this expression evidently vanish because they contain

$$
\left(u_{2}^{+} u_{3}^{+}\right) \delta^{(2,-2)}\left(u_{2}, u_{3}\right) \rightarrow 0
$$

Integrating by parts with respect to the derivative $D_{2}^{--}$in the remaining terms we see that the only expression which does not contain the vanishing product (B.11) is

$$
\begin{array}{rl}
-2 i C_{2} \int d^{8} \theta d u_{2} & d u_{3} \int \frac{d^{6} p}{(2 \pi)^{6}} \frac{d^{6} k}{(2 \pi)^{6}} \frac{1}{k^{4}(k+p)^{2}} \\
& \times V_{\text {linear }}^{--A}\left(-p, \theta, u_{2}\right) \boldsymbol{F}_{\text {linear }}^{++A}\left(p, \theta, u_{2}\right)\left(u_{2}^{-} u_{3}^{+}\right)^{2} \delta^{(2,-2)}\left(u_{2}, u_{3}\right) .
\end{array}
$$

After calculating the harmonic integrals, this expression takes the form

$$
-2 i C_{2} \int d^{8} \theta d u \int \frac{d^{6} p}{(2 \pi)^{6}} \frac{d^{6} k}{(2 \pi)^{6}} \frac{1}{k^{4}(k+p)^{2}} \boldsymbol{V}_{\text {linear }}^{--A}(-p, \theta, u) \boldsymbol{F}_{\text {linear }}^{++A}(p, \theta, u) .
$$

The momentum integral in this expression is calculated in the Euclidean space after the standard Wick rotation with the help of dimensional reduction. Clearly, we are interested in its divergent part only,

$$
\int \frac{d^{6} k}{(2 \pi)^{6}} \frac{1}{k^{4}(k+p)^{2}} \rightarrow-i \int \frac{d^{D} K}{(2 \pi)^{D}} \frac{1}{K^{6}}+\text { finite terms }=-\frac{i}{\varepsilon(4 \pi)^{3}}+\text { finite terms }
$$

where the capital letter $K$ denotes the Euclidean loop momentum.

Thus, in the coordinate representation we obtain the following divergent contribution coming from the supergraph (1) in figure 1:

$$
[\operatorname{diagram}(1)]_{\infty}=-\frac{2 C_{2}}{\varepsilon(4 \pi)^{3}} \int d^{14} z d u \boldsymbol{V}_{\text {linear }}^{--A} \boldsymbol{F}_{\text {linear }}^{++A}=-\frac{4 C_{2}}{\varepsilon(4 \pi)^{3}} \operatorname{tr} \int d \zeta^{(-4)} d u\left(\boldsymbol{F}_{\text {linear }}^{++}\right)^{2}
$$

Now, let us calculate the contribution of the tadpole supergraph (2) in figure 1. The relevant vertex is also obtained from the expression (4.5) and is composed out of the terms 
quadratic in the background gauge superfield $\boldsymbol{V}^{++}$and in the background bridge $\boldsymbol{b}$,

$$
\begin{aligned}
\pm & \frac{1}{2 g_{0}^{2}} \operatorname{tr} \int d^{14} z d u\left\{i \partial^{2} v^{++}\left[\boldsymbol{V}_{\text {quadratic }}^{--}, D^{--} v^{++}\right]+i \partial^{2} v^{++} D^{--}\left[\boldsymbol{V}_{\text {quadratic }}^{--}, v^{++}\right]\right. \\
& -\partial^{2} v^{++}\left[\boldsymbol{V}_{\text {linear }}^{--},\left[\boldsymbol{V}_{\text {linear }}^{--}, v^{++}\right]\right]-\frac{1}{4}\left(\left[\boldsymbol{V}_{\text {linear }}^{--}, D^{--} v^{++}\right]+D^{--}\left[\boldsymbol{V}_{\text {linear }}^{--}, v^{++}\right]\right)\left(D^{+}\right)^{4} \\
& \left.\times\left(\left[\boldsymbol{V}_{\text {linear }}^{--}, D^{--} v^{++}\right]+D^{--}\left[\boldsymbol{V}_{\text {linear }}^{--}, v^{++}\right]\right)\right\} \pm \frac{1}{2 g_{0}^{2}} \operatorname{tr} \int d^{14} z d u_{1} d u_{2} \frac{1}{\left(u_{1}^{+} u_{2}^{+}\right)^{2}} \\
& \times\left\{\left[\boldsymbol{b}_{1}-\boldsymbol{b}_{2}, v_{2}^{++}\right]\left[\boldsymbol{F}_{\text {linear }, 1}^{++}, D^{--} v_{1}^{++}\right]-i v_{2}^{++}\left[\boldsymbol{F}_{\text {quadratic }, 1}^{++}, D^{--} v_{1}^{++}\right]\right\}
\end{aligned}
$$

where $\boldsymbol{V}_{\text {quadratic }}^{--}$and $\boldsymbol{F}_{\text {quadratic }}^{++}$are those parts of $\boldsymbol{V}^{--}$and $\boldsymbol{F}^{++}$which are quadratic in $\boldsymbol{V}^{++}$. Constructing the corresponding expression for the contribution of the supergraph (2), we see that all terms in it vanish either because the number of spinor derivatives acting on the anticommuting $\delta$-function at the coincident arguments is less than 8 (for all terms without $\left(D^{+}\right)^{4}$ in eq. (B.16)), or because of the presence of $\left.\left(u_{1}^{+} u_{2}^{+}\right)\right|_{u_{1}=u_{2}}=0$ (for the terms containing $\left.\left(D^{+}\right)^{4}\right)$. Therefore, the supergraph (2) in figure 1 does not contribute to the divergent part of the one-loop effective action.

Thus, the one-loop divergences coming from the supergraphs (1) and (2) in figure 1 are given by eq. (B.15). However, the gauge superfield $\boldsymbol{V}^{++}$is dimensionless, so that the oneloop divergences are also present in supergraphs with an arbitrary number of the external gauge legs. The general result for the divergent part of the one-loop effective action can be restored by resorting to the manifest gauge invariance of the effective action $\Gamma\left[\boldsymbol{V}^{++}\right] .{ }^{7}$ The gauge invariant expression which in the lowest approximation yields eq. (B.15) is evidently given just by eq. (4.6).

Open Access. This article is distributed under the terms of the Creative Commons Attribution License (CC-BY 4.0), which permits any use, distribution and reproduction in any medium, provided the original author(s) and source are credited.

\section{References}

[1] E.S. Fradkin and A.A. Tseytlin, Quantum Properties of Higher Dimensional and Dimensionally Reduced Supersymmetric Theories, Nucl. Phys. B 227 (1983) 252 [InSPIRE].

[2] N. Marcus and A. Sagnotti, A Test of Finiteness Predictions for Supersymmetric Theories, Phys. Lett. B 135 (1984) 85 [inSPIRE].

[3] N. Marcus and A. Sagnotti, The Ultraviolet Behavior of $N=4$ Yang-Mills and the Power Counting of Extended Superspace, Nucl. Phys. B 256 (1985) 77 [InSPIRE].

[4] P.S. Howe and K.S. Stelle, Ultraviolet Divergences in Higher Dimensional Supersymmetric Yang-Mills Theories, Phys. Lett. B 137 (1984) 175 [INSPIRE].

[5] P.S. Howe and K.S. Stelle, Supersymmetry counterterms revisited, Phys. Lett. B 554 (2003) 190 [hep-th/0211279] [INSPIRE].

\footnotetext{
${ }^{7}$ Note that in the case of using the background field method one-loop contributions generated by the supergraphs with a gauge loop and by the supergraphs with a ghost loop are gauge invariant separately.
} 
[6] G. Bossard, P.S. Howe and K.S. Stelle, The Ultra-violet question in maximally supersymmetric field theories, Gen. Rel. Grav. 41 (2009) 919 [arXiv:0901.4661] [InSPIRE].

[7] G. Bossard, P.S. Howe and K.S. Stelle, A Note on the UV behaviour of maximally supersymmetric Yang-Mills theories, Phys. Lett. B 682 (2009) 137 [arXiv:0908.3883] [INSPIRE].

[8] L.V. Bork, D.I. Kazakov, M.V. Kompaniets, D.M. Tolkachev and D.E. Vlasenko, Divergences in maximal supersymmetric Yang-Mills theories in diverse dimensions, JHEP 11 (2015) 059 [arXiv: 1508.05570] [INSPIRE].

[9] A. Pais and G.E. Uhlenbeck, On Field theories with nonlocalized action, Phys. Rev. 79 (1950) 145 [INSPIRE].

[10] A.A. Starobinsky, A New Type of Isotropic Cosmological Models Without Singularity, Phys. Lett. B 91 (1980) 99 [Adv. Ser. Astrophys. Cosmol. 3 (1987) 130] [INSPIRE].

[11] S.W. Hawking, Who's Afraid Of (Higher Derivative) Ghosts?, in Quantum Field Theory and Quantum Statistics, I.A. Batalin, C.J. Isham and G.A. Vilkovisky eds., vol. 2, pp. 129-139, Adam Hilger Publishing (1986) [INSPIRE].

[12] K.S. Stelle, Renormalization of Higher Derivative Quantum Gravity, Phys. Rev. D 16 (1977) 953 [INSPIRE].

[13] E.S. Fradkin and A.A. Tseytlin, Renormalizable asymptotically free quantum theory of gravity, Nucl. Phys. B 201 (1982) 469 [inSPIRE].

[14] I.L. Buchbinder, S.D. Odintsov and I.L. Shapiro, Effective Action in Quantum Gravity, IOP, Bristol and Philadelphia, U.K. (1992) [InSPIRE].

[15] B. Grinstein and D. O'Connell, One-Loop Renormalization of Lee-Wick Gauge Theory, Phys. Rev. D 78 (2008) 105005 [arXiv:0801.4034] [INSPIRE].

[16] M. Beccaria and A.A. Tseytlin, Conformal anomaly c-coefficients of superconformal $6 d$ theories, JHEP 01 (2016) 001 [arXiv:1510.02685] [INSPIRE].

[17] H. Osborn and A. Stergiou, $C_{T}$ for non-unitary CFTs in higher dimensions, JHEP 06 (2016) 079 [arXiv: 1603.07307] [INSPIRE].

[18] S.M. Kuzenko, J. Novak and S. Theisen, New superconformal multiplets and higher derivative invariants in six dimensions, Nucl. Phys. B 925 (2017) 348 [arXiv:1707.04445] [INSPIRE].

[19] A.R.R. Castellanos, F. Sobreira, I.L. Shapiro and A.A. Starobinsky, On higher derivative corrections to the $R+R^{2}$ inflationary model, JCAP 12 (2018) 007 [arXiv:1810.07787] [INSPIRE].

[20] D. Anselmi, The quest for purely virtual quanta: fakeons versus Feynman-Wheeler particles, JHEP 03 (2020) 142 [arXiv: 2001.01942] [INSPIRE].

[21] A. Smilga, Ultraviolet divergences in non-renormalizable supersymmetric theories, Phys. Part. Nucl. Lett. 14 (2017) 245 [arXiv:1603.06811] [INSPIRE].

[22] L. Casarin, On higher-derivative gauge theories, arXiv:1710.08021 [INSPIRE].

[23] A.A. Slavnov, Invariant regularization of nonlinear chiral theories, Nucl. Phys. B 31 (1971) 301 [INSPIRE].

[24] A.A. Slavnov, Invariant regularization of gauge theories, Theor. Math. Phys. 13 (1972) 1064 [INSPIRE]. 
[25] V.K. Krivoshchekov, Invariant Regularizations for Supersymmetric Gauge Theories, Theor. Math. Phys. 36 (1978) 291 [INSPIRE].

[26] P.C. West, Higher Derivative Regulation of Supersymmetric Theories, Nucl. Phys. B 268 (1986) 113 [INSPIRE].

[27] I.L. Buchbinder and K.V. Stepanyantz, The higher derivative regularization and quantum corrections in $N=2$ supersymmetric theories, Nucl. Phys. B $\mathbf{8 8 3}$ (2014) 20 [arXiv: 1402.5309] [INSPIRE].

[28] I.L. Buchbinder, N.G. Pletnev and K.V. Stepanyantz, Manifestly $N=2$ supersymmetric regularization for $N=2$ supersymmetric field theories, Phys. Lett. B 751 (2015) 434 [arXiv: 1509.08055] [INSPIRE].

[29] S.J. Gates, M.T. Grisaru, M. Roček and W. Siegel, Superspace Or One Thousand and One Lessons in Supersymmetry, Front. Phys. 58 (1983) 1 [hep-th/0108200] [INSPIRE].

[30] P.C. West, Introduction to supersymmetry and supergravity, World Scientific, Singapore, Singapore (1990) [INSPIRE].

[31] I.L. Buchbinder and S.M. Kuzenko, Ideas and methods of supersymmetry and supergravity: Or a walk through superspace, IOP, Bristol, U.K. (1998) [INSPIRE].

[32] K. Stepanyantz, The higher covariant derivative regularization as a tool for revealing the structure of quantum corrections in supersymmetric gauge theories, Proc. Steklov Inst. Math. 309 (2020) 284 [arXiv: 1910.03242] [INSPIRE].

[33] K.V. Stepanyantz, The NSVZ $\beta$-function for theories regularized by higher covariant derivatives: the all-loop sum of matter and ghost singularities, JHEP 01 (2020) 192 [arXiv: 1912.12589] [INSPIRE].

[34] E.A. Ivanov, A.V. Smilga and B.M. Zupnik, Renormalizable supersymmetric gauge theory in six dimensions, Nucl. Phys. B 726 (2005) 131 [hep-th/0505082] [InSPIRE].

[35] L. Casarin and A.A. Tseytlin, One-loop $\beta$-functions in 4-derivative gauge theory in 6 dimensions, JHEP 08 (2019) 159 [arXiv:1907.02501] [INSPIRE].

[36] I.L. Buchbinder, E.A. Ivanov, B.S. Merzlikin and K.V. Stepanyantz, The renormalization structure of $6 D, \mathcal{N}=(1,0)$ supersymmetric higher-derivative gauge theory, arXiv : 2007.02843 [INSPIRE].

[37] A.V. Smilga, Chiral anomalies in higher-derivative supersymmetric 6D theories, Phys. Lett. B 647 (2007) 298 [hep-th/0606139] [INSPIRE].

[38] P.S. Howe, K.S. Stelle and P.C. West, $N=1 d=6$ harmonic superspace, Class. Quant. Grav. 2 (1985) 815 [INSPIRE].

[39] B.M. Zupnik, Six-dimensional Supergauge Theories in the Harmonic Superspace, Sov. J. Nucl. Phys. 44 (1986) 512 [inSPIRE].

[40] G. Bossard, E. Ivanov and A. Smilga, Ultraviolet behavior of 6D supersymmetric Yang-Mills theories and harmonic superspace, JHEP 12 (2015) 085 [arXiv:1509.08027] [INSPIRE].

[41] A. Galperin, E. Ivanov, V. Ogievetsky and E. Sokatchev, Harmonic superspace: key to $N=2$ supersymmetry theories, JETP Lett. 40 (1984) 912 [INSPIRE].

[42] A. Galperin, E. Ivanov, S. Kalitsyn, V. Ogievetsky and E. Sokatchev, Unconstrained $N=2$ Matter, Yang-Mills and Supergravity Theories in Harmonic Superspace, Class. Quant. Grav. 1 (1984) 469 [Erratum ibid. 2 (1985) 127] [INSPIRE]. 
[43] A.S. Galperin, E.A. Ivanov, V.I. Ogievetsky and E.S. Sokatchev, Harmonic superspace, Cambridge University Press, Cambridge, U.K. (2001) [DOI] [INSPIRE].

[44] I.L. Buchbinder, E.A. Ivanov, B.S. Merzlikin and K.V. Stepanyantz, One-loop divergences in the $6 D, \mathcal{N}=(1,0)$ abelian gauge theory, Phys. Lett. B 763 (2016) 375 [arXiv:1609.00975] [INSPIRE].

[45] I.L. Buchbinder, E.A. Ivanov, B.S. Merzlikin and K.V. Stepanyantz, One-loop divergences in $6 D, \mathcal{N}=(1,0)$ SYM theory, JHEP 01 (2017) 128 [arXiv:1612.03190] [InSPIRE].

[46] I.L. Buchbinder, E.A. Ivanov, B.S. Merzlikin and K.V. Stepanyantz, Supergraph analysis of the one-loop divergences in $6 D, \mathcal{N}=(1,0)$ and $\mathcal{N}=(1,1)$ gauge theories, Nucl. Phys. $B \mathbf{9 2 1}$ (2017) 127 [arXiv: 1704.02530] [INSPIRE].

[47] I.L. Buchbinder, E.A. Ivanov, B.S. Merzlikin and K.V. Stepanyantz, On the two-loop divergences of the 2-point hypermultiplet supergraphs for $6 D, \mathcal{N}=(1,1) S Y M$ theory, Phys. Lett. B 778 (2018) 252 [arXiv:1711.11514] [INSPIRE].

[48] I.L. Buchbinder, E.A. Ivanov and B.S. Merzlikin, Leading low-energy effective action in $6 D$, $\mathcal{N}=(1,1)$ SYM theory, JHEP 09 (2018) 039 [arXiv: 1711.03302] [INSPIRE].

[49] I.L. Buchbinder, E. Ivanov, B. Merzlikin and K. Stepanyantz, Harmonic Superspace Approach to the Effective Action in Six-Dimensional Supersymmetric Gauge Theories, Symmetry 11 (2019) 68 [arXiv:1812.02681] [INSPIRE].

[50] I.L. Buchbinder, E.A. Ivanov, B.S. Merzlikin and K.V. Stepanyantz, Gauge dependence of the one-loop divergences in $6 D, \mathcal{N}=(1,0)$ abelian theory, Nucl. Phys. B 936 (2018) 638 [arXiv: 1808.08446] [INSPIRE].

[51] B.M. Zupnik, The Action of the Supersymmetric $N=2$ Gauge Theory in Harmonic Superspace, Phys. Lett. B 183 (1987) 175 [InSPIRE].

[52] B.S. DeWitt, Dynamical theory of groups and fields, Conf. Proc. C 630701 (1964) 585 [INSPIRE].

[53] L.F. Abbott, The Background Field Method Beyond One Loop, Nucl. Phys. B 185 (1981) 189 [INSPIRE].

[54] L.F. Abbott, Introduction to the Background Field Method, Acta Phys. Polon. B 13 (1982) 33 [INSPIRE].

[55] I.L. Buchbinder, E.I. Buchbinder, S.M. Kuzenko and B.A. Ovrut, The Background field method for $N=2$ superYang-Mills theories in harmonic superspace, Phys. Lett. B 417 (1998) 61 [hep-th/9704214] [INSPIRE].

[56] E.I. Buchbinder, B.A. Ovrut, I.L. Buchbinder, E.A. Ivanov and S.M. Kuzenko, Low-energy effective action in $N=2$ supersymmetric field theories, Phys. Part. Nucl. 32 (2001) 641 [INSPIRE].

[57] E.A. Ivanov and A.V. Smilga, Conformal properties of hypermultiplet actions in six dimensions, Phys. Lett. B 637 (2006) 374 [hep-th/0510273] [INSPIRE].

[58] I.L. Buchbinder and N.G. Pletnev, Effective actions in $\mathcal{N}=1$, D5 supersymmetric gauge theories: harmonic superspace approach, JHEP 11 (2015) 130 [arXiv:1510.02563] [INSPIRE]. 